\title{
IQ Imbalance Compensation: A Semi-Blind Method for OFDM Systems in Fast Fading Channels
}

\author{
Lanlan He, Shaodan Ma, Yik-Chung Wu, Tung-Sang Ng \\ Department of Electrical and Electronic Engineering, The University of Hong Kong \\ Email:\{llhe, sdma, ycwu, tsng\}@eee.hku.hk
}

\begin{abstract}
In this paper, an orthogonal frequency division multiplexing (OFDM) system operating in a fast fading environment modeled by a doubly selective channel (DSC) is considered. The paper first reformulates a commonly adopted system model using the generalized complex exponential basis expansion technique. The resulting model enables the IQ imbalance and DSC to be estimated in the time domain with a small number of scattered pilots within an OFDM symbol. A joint estimation and compensation scheme is then proposed which compensates all the inter-carrier interference terms. Simulation results show that the proposed compensation method achieves better symbol error rate performance than previous proposed methods.
\end{abstract}

\section{INTRODUCTION}

In recent developments of mobile TV technologies [1], [2], and 4G mobile communications systems [3], high speed movement of mobile terminals, e.g., high speed trains, has attracted lots of attention. High speed mobile terminals cause Doppler spread and result in time-varying multi-path channels, particularly in broadband OFDM systems. These channels are often represented by doubly selective channels (DSCs). Representative works on channel estimation and inter-carrier interference (ICI) compensation for DSCs have been given in [4]-[6], where basis expansion models (BEMs) are often used to approximate the DSCs. Moreover, a pilot cluster structure, in which each cluster contains only one non-zero pilot in the middle, is widely adopted for channel estimation.

With continued fast development of CMOS technologies, direct-conversion architectures which directly convert radio frequency (RF) signals to baseband signals and vice versa, become attractive. However, direct conversion tends to suffer from impairments, e.g., non-ideal analog filters, which introduces in-phase and quadrature-phase (IQ) imbalance. For slow fading channels, IQ imbalance compensation has been extensively studied [7]-[13]. On the other hand, when the system is operating over DSCs, the energy of data on one subcarrier not only spreads over adjacent subcarriers, but also becomes mirrored interference within the OFDM symbol. The methods [4]-[6], [10]-[12] proposed for either DSCs or IQ imbalance compensation will therefore perform poorly. Recently, in [14], the effects of both IQ imbalance and the DSC was quantified, a general mathematical model was developed, and IQ imbalance compensation algorithms were also proposed. Joint estimation of the IQ imbalance and DSC was also addressed by exploiting scattered pilots. The combined effects of the IQ imbalance and DSC are estimated in the frequency domain, where the number of parameters to be estimated is at least the square of the number of subcarriers, i.e., the square of the number of observations for estimation. To make the estimation and compensation feasible, only the IQ imbalance

The work was supported in part by the GRF, Project No. HKU 7154/08E and the HKU Seed Funding Programme, Project No. 200811159094. and DSC parameters related to the most significant ICI terms are estimated, and half of the ICI terms are left uncompensated and are treated as noise.

In this paper, a different approach from that of [14] is proposed. To be specific, the system model is reformulated using the generalized complex exponential basis expansion model (GCE-BEM), so that the IQ imbalance and DSC can be estimated in the time-domain with a small number of scattered pilots. A joint compensation scheme is then proposed, which compensates all the ICI terms. Simulation results show that the proposed compensation method achieves better symbol error rate (SER) performance than the method in [14].

Notation: Boldface uppercase and lowercase letters are used for matrices and vectors respectively. Superscripts $*, T$ and $H$ denote conjugate, transpose and Hermitian respectively. The symbol $\mathbf{I}_{N}$ denotes the $N \times N$ identity matrix. The symbol $\operatorname{diag}\{\mathbf{x}\}$ signifies the diagonal matrix with vector $\mathbf{x}$ on its diagonal. $\Re\{\cdot\}$ and $\Im\{\cdot\}$ are the real and imaginary parts respectively. The matrix $\mathbf{F}$ denotes the fast Fourier transform (FFT) matrix with $[\mathbf{F}]_{m, n}=\frac{1}{\sqrt{N}} e^{-j 2 \pi m n / N}$. $\lceil a\rceil$ rounds $a$ to the nearest integer greater than or equal to $a$.

\section{SySTEM MODEL}

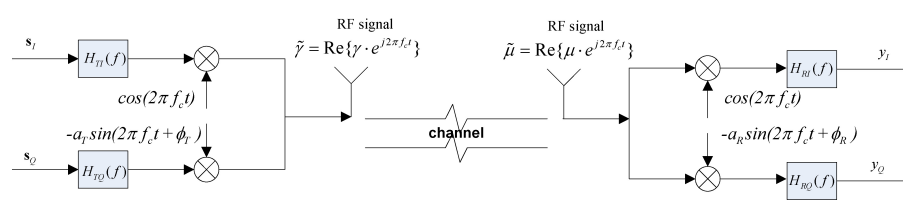

Fig. 1. Block diagram of the transmitter and receiver with IQ imbalance.

\section{A. Transmitted OFDM signal with scattered pilots}

In an OFDM system, the source data in the frequency domain $\mathbf{x}=[x(0), \cdots, x(N-1)]^{T}$ is modulated onto $N$ parallel subcarriers to obtain the time domain signal $\mathbf{s}=\mathbf{F}^{H} \mathbf{x}$. In general, the elements of $\mathrm{x}$ can be categorized into

$$
x(k)=\left\{\begin{array}{lll}
x_{p}(k) & \forall & k \in I_{p} \\
x_{d}(k) & \forall & k \in I_{d}
\end{array}\right.
$$

where $I_{p}$ is the index set of subcarriers allocated for pilot symbols with $N_{p}$ elements, and $I_{d}$ is the index set of data subcarriers with $N_{d}$ elements. Notice that $N_{p}+N_{d}=N$. From (1), we have $\mathbf{x}=\mathbf{E}_{d} \mathbf{x}_{d}+\mathbf{E}_{p} \mathbf{x}_{p}$, where $\mathbf{E}_{d}$ and $\mathbf{E}_{p}$ denote matrices collecting columns of $\mathbf{I}_{N}$ corresponding to $I_{d}$ and $I_{p}$ respectively, and $\mathbf{x}_{p}$ and $\mathbf{x}_{d}$ denote pilots and data vectors respectively.

A cyclic prefix (CP) of length $L_{c p}$ is inserted at the beginning of the time domain OFDM signal $\mathrm{s}$ to prevent intersymbol interference (ISI). The baseband signal is then converted to a radio frequency signal through a direct conversion transmitter. 
The direct conversion transmitter is shown in Fig. 1, where the frequency independent IQ imbalance is characterized by an amplitude mismatch $\alpha_{T}$ and a phase mismatch $\phi_{T}$, while the frequency dependent IQ imbalance is modeled by two filters in $\mathrm{I}$ and $\mathrm{Q}$ branches with frequency responses as $H_{T I}(f)$ and $H_{T Q}(f)$ respectively. According to [10] and following derivations in [12], the time domain transmitter IQ imbalance coefficients are given by

$$
\begin{aligned}
g_{T I}(n) & =\left.\mathcal{F}^{-1}\left\{\frac{H_{T I}(f)+\alpha_{T} e^{-j \phi_{T}} H_{T Q}(f)}{2}\right\}\right|_{t=n T_{s}}(2) \\
\text { and } \quad g_{T Q}(n) & =\left.\mathcal{F}^{-1}\left\{\frac{H_{T I}(f)-\alpha_{T} e^{j \phi_{T}} H_{T Q}(f)}{2}\right\}\right|_{t=n T_{s}} \text { (3) }
\end{aligned}
$$

where $\mathcal{F}^{-1}$ denotes the inverse Fourier transformation, $T_{s}$ is the sample interval and $n=0, \cdots, L_{t}-1$ with $L_{t}$ being the length of the time domain transmitter IQ imbalance filters.

We consider a Rayleigh-distributed DSC that has $L_{h}$ independent taps with the average power of the $l^{\text {th }}$ tap denoted by $\sigma_{l}^{2}$. The auto-correlation of the $l^{\text {th }}$ tap coefficient follows the classical Jakes' model [5] given by $\mathbb{E}\left\{h_{l}\left(m T_{s}\right) h_{l}^{*}\left(n T_{s}\right)\right\}=\sigma_{l}^{2} J_{0}\left(2 \pi f_{d}(n-m) T_{s}\right)$, where $J_{0}(\cdot)$ is the zero-order Bessel function of the first kind and $f_{d}$ denotes the Doppler spread normalized by the subcarrier spacing. At the direct-conversion receiver side, similarly to (2) and (3), the time domain receiver IQ imbalance coefficients are given by $g_{R I}(n)=\left.\mathcal{F}^{-1}\left\{\left(H_{R I}(f)+\alpha_{R} e^{-j \phi_{R}} H_{R Q}(f)\right) / 2\right\}\right|_{t=n T_{s}}$ and $g_{R Q}(n)=\left.\mathcal{F}^{-1}\left\{\left(H_{R I}(f)-\alpha_{R} e^{j \phi_{R}} H_{R Q}(f)\right) / 2\right\}\right|_{t=n T_{s}}$, $\left(n=0, \cdots, L_{r}-1\right)$, respectively. Here, $\alpha_{R}$ and $\phi_{R}$ respectively denote the amplitude and phase mismatches, $H_{R I}(f)$ and $H_{R Q}(f)$ denote the frequency responses of two filters in the $\mathrm{I}$ and $\mathrm{Q}$ branches respectively, and $L_{r}$ is the length of the time domain receiver IQ imbalance filters. The received signal after transformation into the frequency domain $\overline{\mathbf{y}}=[\bar{y}(0), \cdots, \bar{y}(N-1)]^{T}$ is then given by

$$
\begin{aligned}
& \overline{\mathbf{y}}=\mathbf{F G}_{R I} \mathbf{H} \mathbb{I}_{L} \mathbf{G}_{T I} \mathbf{F}^{H} \mathbf{x}+\mathbf{F} \mathbf{G}_{R I} \mathbf{H I}_{L} \mathbf{G}_{T Q}\left(\mathbf{F}^{H} \mathbf{x}\right)^{*} \\
& +\mathbf{F G}_{R Q} \mathbf{H}^{*} \mathbb{I}_{L} \mathbf{G}_{T I}^{*}\left(\mathbf{F}^{H} \mathbf{x}\right)^{*}+\mathbf{F G}_{R Q} \mathbf{H}^{*} \mathbb{I}_{L} \mathbf{G}_{T Q}^{*} \mathbf{F}^{H} \mathbf{x} \\
& +\underbrace{\mathbf{F G} \mathbf{G}_{R I} \mathbf{w}+\mathbf{F G}_{R Q} \mathbf{w}^{*}}_{\triangleq \overline{\mathbf{w}}},
\end{aligned}
$$

where $\mathbf{G}_{R I}$ and $\mathbf{G}_{R Q}$ are $N \times\left(N+L_{r}-1\right)$ Toeplitz matrices with the first rows being $\left[g_{R I}\left(L_{r}-1\right), \cdots, g_{R I}(0), 0, \cdots, 0\right]^{T}$ and $\left[g_{R Q}\left(L_{r}-1\right), \cdots, g_{R Q}(0), 0, \cdots, 0\right]^{T}$ respectively, $\mathbf{G}_{T I}$ and $\mathbf{G}_{T Q}$ are $N \times N$ circular matrices with the first columns being $\left[g_{T I}(0), g_{T I}(1), \cdots, g_{T I}\left(L_{t}-1\right), 0, \cdots, 0\right]^{T}$ and $\left[g_{T Q}(0), g_{T Q}(1), \cdots, g_{T Q}\left(L_{t}-1\right), 0, \cdots, 0\right]^{T}$ respectively, $\mathbf{w}$ denotes an additive white Gaussian noise vector $\mathbf{w}=\left[w\left(-\left(L_{r}-1\right)\right), \cdots, w(0), \cdots, w(N-1)\right]^{T}$, and $\mathbf{H}$ is an $\left(N+L_{r}-1\right) \times\left(N+L_{r}+L_{h}-2\right)$ matrix given by

$$
\mathbf{H}=\left[\begin{array}{c}
h\left(L_{h}-1,-\left(L_{r}-1\right)\right) \cdots h\left(0,-\left(L_{r}-1\right)\right) \\
h\left(L_{h}-1,1\right) \cdots \cdots \\
\ldots \ldots \cdots \cdots \\
h\left(L_{h}-1, N-1\right) \cdots h(0, N-1)
\end{array}\right]
$$

with $h(l, n)$ being the sample of the $l^{\text {th }}$ tap at time $n T_{s}\left(T_{s}\right.$ is omitted for notational simplicity). In (4), $\mathbb{I}_{L}=\left[\mathbf{E}_{L}, \mathbf{I}_{N}\right]^{T}$ with $\mathbf{E}_{L}$ being the last $L \triangleq L_{r}+L_{h}-2$ columns of the identity matrix $\mathbf{I}_{N}$, and it represents the effect of the CP.

\section{B. Reformulation with basis expansion model}

Notice that the channel matrix $\mathbf{H}$ is completely characterized by $\mathbf{h} \triangleq\left[\mathbf{h}_{0}^{T}, \cdots, \mathbf{h}_{L-1}^{T}\right]^{T}$ with $\mathbf{h}_{l} \triangleq\left[h\left(l,-\left(L_{r}-\right.\right.\right.$
1)), $\cdots, h(l, N-1)]^{T}$ being the channel coefficients of the $l^{t h}$ tap over interval $\left[-\left(L_{r}-1\right), \cdots, N-1\right]$. The number of unknown channel parameters is $\left(N+L_{r}-1\right) L$, which is much larger than the number of received samples. This makes least squares and maximum likelihood (ML) estimation impossible.

To reduce the number of unknown parameters, the generalized complex exponential basis expansion model (GCE-BEM) [5] is adopted to represent the DSC. With GCE-BEM, the matrix $\mathbf{H}$ in (5) can be approximated by

$$
\mathbf{H} \cong \sum_{m=-\rho}^{\rho} \boldsymbol{\Gamma}(m) \mathbf{H}_{m}
$$

where $\rho=\left\lceil G N f_{d} T_{s}\right\rceil$ with $G$ being the oversampling factor in Doppler domain,

$$
\boldsymbol{\Gamma}(m)=\operatorname{diag}\left\{e^{j 2 \pi m\left(-\left(L_{r}-1\right)\right) / G N}, \cdots, e^{j 2 \pi m(N-1) / G N}\right\}
$$

and

$$
\mathbf{H}_{m}=\left[\begin{array}{cccc}
h_{m}\left(L_{h}-1\right) & \ldots & h_{m}(0) & \ldots \\
\ldots & \ldots & \ldots & \ldots \\
\mathbf{0} & h_{m}\left(L_{h}-1\right) & \ldots & h_{m}(0)
\end{array}\right]
$$

with $h_{m}(l)$ denoting the BEM coefficient. Based on the GCE$\mathrm{BEM}$, the total number of channel parameters is significantly reduced from $\left(N+L_{r}-1\right) L_{h}$ to $(2 \rho+1) L_{h}$. Substituting (6) into (4), it follows that

$$
\begin{aligned}
\overline{\mathbf{y}} & \cong \sum_{m=-\rho}^{\rho}\left(\mathbf{F} \mathbf{G}_{R I} \boldsymbol{\Gamma}(m) \mathbf{H}_{m} \mathbb{I}_{L} \mathbf{G}_{T I} \mathbf{F}^{H} \mathbf{x}\right. \\
& +\mathbf{F G}_{R I} \boldsymbol{\Gamma}(m) \mathbf{H}_{m} \mathbb{I}_{L} \mathbf{G}_{T Q}\left(\mathbf{F}^{H} \mathbf{x}\right)^{*} \\
& +\mathbf{F} \mathbf{G}_{R Q} \boldsymbol{\Gamma}(m) \mathbf{H}_{-m}^{*} \mathbb{I}_{L} \mathbf{G}_{T I}^{*}\left(\mathbf{F}^{H} \mathbf{x}\right)^{*} \\
& \left.+\mathbf{F G}_{R Q} \boldsymbol{\Gamma}(m) \mathbf{H}_{-m}^{*} \mathbb{I}_{L} \mathbf{G}_{T Q}^{*} \mathbf{F}^{H} \mathbf{x}\right)+\overline{\mathbf{w}}
\end{aligned}
$$

where we have also used the fact that $\mathbf{H}^{*} \cong$ $\sum_{m=-\rho}^{\rho} \boldsymbol{\Gamma}(m) \mathbf{H}_{-m}^{*}$.

As can be seen from (9), $\mathbf{G}_{R I}, \mathbf{G}_{T I}, \mathbf{G}_{R Q}$ and $\mathbf{G}_{T Q}$ are all coupled with BEM coefficients, and separately estimating these parameters is difficult. Fortunately, in joint IQ imbalance and channel compensation, only the combined effects of the DSC and IQ imbalance need to be estimated. It is proved (omitted due to space limitation) that the combined effects can be represented by two unknown vectors $\mathbf{u}_{m 1} \triangleq\left[u_{m 1}(0), \cdots, u_{m 1}\left(L_{e}-1\right)\right]^{T}$ and $\mathbf{u}_{m 2} \triangleq$ $\left[u_{m 2}(0), \cdots, u_{m 2}\left(L_{e}-1\right)\right]^{T}$ with $L_{e} \triangleq L_{r}+L_{h}+L_{t}-2$, and (9) can be equivalently written as

$$
\begin{aligned}
\overline{\mathbf{y}} & =\sum_{m=-\rho}^{\rho}\left(\mathbf{F} \boldsymbol{\Phi}(m) \mathbf{F}^{H} \operatorname{diag}\left\{\mathbf{F}_{L_{e}} \mathbf{u}_{m 1}\right\} \mathbf{x}\right. \\
& \left.+\mathbf{F} \boldsymbol{\Phi}(m) \mathbf{F}^{H} \operatorname{diag}\left\{\mathbf{F}_{L_{e}} \mathbf{u}_{m 2}\right\} \mathbf{x}^{\#}\right)+\overline{\mathbf{w}}
\end{aligned}
$$

where $\boldsymbol{\Phi}(m)=\operatorname{diag}\left\{1, e^{j 2 \pi m / G N}, \cdots, e^{j 2 \pi m(N-1) / G N}\right\}$, and $\mathbf{x}^{\#}=\mathbf{F F x} \mathbf{x}^{*}$ is the mirrored version of $\mathbf{x}$, namely the $k^{t h}$ element of $\mathbf{x}^{\#}$ is given by the conjugate of the $(N-k)^{t h}$ element of $\mathbf{x}$.

\section{IQ IMBALANCE AND CHANNEL COMPENSATION WITH SCATTERED PILOTS}

\section{A. Channel estimation}

In order to compensate for the effects of IQ imbalance and channel, we must first estimate the unknown vectors $\mathbf{u}_{m 1}$ and 


$$
\begin{aligned}
& \overline{\mathbf{y}}_{p}=\sum_{m=-\rho}^{\rho}\left(\mathbf{F}_{p} \boldsymbol{\Phi}(m) \mathbf{F}^{H} \operatorname{diag}\left\{\mathbf{E}_{p} \mathbf{x}_{p}\right\} \mathbf{F}_{L_{e}} \mathbf{u}_{m 1}+\mathbf{F}_{p} \boldsymbol{\Phi}(m) \mathbf{F}^{H} \operatorname{diag}\left\{\left(\mathbf{E}_{p} \mathbf{x}_{p}\right)^{\#}\right\} \mathbf{F}_{L_{e}} \mathbf{u}_{m 2}\right) \\
& \underbrace{\sum_{m=-\rho}^{\rho}\left(\mathbf{F}_{p} \boldsymbol{\Phi}(m) \mathbf{F}^{H} \operatorname{diag}\left\{\mathbf{E}_{d} \mathbf{x}_{d}\right\} \mathbf{F}_{L_{e}} \mathbf{u}_{m 1}+\mathbf{F}_{p} \boldsymbol{\Phi}(m) \mathbf{F}^{H} \operatorname{diag}\left\{\left(\mathbf{E}_{d} \mathbf{x}_{d}\right)^{\#}\right\} \mathbf{F}_{L_{e}} \mathbf{u}_{m 2}\right)+\mathbf{F}_{p} \mathbf{G}_{R I} \mathbf{w}+\mathbf{F}_{p} \mathbf{G}_{R Q} \mathbf{w}^{*}}_{\triangleq \boldsymbol{\delta}} \\
& \overline{\mathbf{y}}=\underbrace{\sum_{m=-\rho}^{\rho} \mathbf{F} \boldsymbol{\Phi}(m) \mathbf{F}^{H} \operatorname{diag}\left\{\mathbf{F}_{L_{e}} \mathbf{u}_{m 1}\right\} \mathbf{E}_{d} \mathbf{x}_{d}}_{\triangleq \mathbf{B}_{1}[\mathbf{u}]}+\underbrace{\sum_{m=-\rho}^{\rho} \mathbf{F} \boldsymbol{\Phi}(m) \mathbf{F}^{H} \operatorname{diag}\left\{\mathbf{F}_{L_{e}} \mathbf{u}_{m 2}\right\} \mathbf{F F} \mathbf{E}_{d}^{*} \mathbf{x}_{d}^{*}}_{\triangleq \mathbf{B}_{2}[\mathbf{u}]} \\
& +\underbrace{\sum_{m=-\rho}^{\rho} \mathbf{F} \boldsymbol{\Phi}(m) \mathbf{F}^{H} \operatorname{diag}\left\{\mathbf{F}_{L_{e}} \mathbf{u}_{m 1}\right\} \mathbf{E}_{p} \mathbf{x}_{p}+\sum_{m=-\rho}^{\rho} \mathbf{F} \boldsymbol{\Phi}(m) \mathbf{F}^{H} \operatorname{diag}\left\{\mathbf{F}_{L_{e}} \mathbf{u}_{m 2}\right\} \mathbf{F} \mathbf{F} \mathbf{E}_{p}^{*} \mathbf{x}_{p}^{*}}_{\triangleq \boldsymbol{\eta}[\mathbf{u}]}+\overline{\mathbf{w}}
\end{aligned}
$$

$\mathbf{u}_{m 2}$. As mentioned earlier in Section II, pilots are inserted in each OFDM symbol. Specifically, pilot subcarriers are grouped into pairs and the $k^{\text {th }}$ pilot pair contains two pilot clusters locating on subcarrier sets $\left[k-\lambda_{1}, \cdots, k+\lambda_{2}\right]$ and $[N-(k+$ $\left.\left.\lambda_{2}\right), \cdots, N-\left(k-\lambda_{1}\right)\right]$ respectively (with one cluster being the mirror of the other one). Each cluster may contain only one non-zero pilot in the middle [6], or multiple non-zero pilots [14].

Reversing the position of data and $\mathbf{u}_{m 1}, \mathbf{u}_{m 2}$ in (10) gives

$$
\begin{aligned}
\overline{\mathbf{y}} & =\sum_{m=-\rho}^{\rho}\left(\mathbf{F} \boldsymbol{\Phi}(m) \mathbf{F}^{H} \operatorname{diag}\{\mathbf{x}\} \mathbf{F}_{L_{e}} \mathbf{u}_{m 1}\right. \\
& \left.+\mathbf{F} \boldsymbol{\Phi}(m) \mathbf{F}^{H} \operatorname{diag}\left\{\mathbf{x}^{\#}\right\} \mathbf{F}_{L_{e}} \mathbf{u}_{m 2}\right)+\overline{\mathbf{w}} .
\end{aligned}
$$

Based on $\mathbf{x}=\mathbf{E}_{d} \mathbf{x}_{d}+\mathbf{E}_{p} \mathbf{x}_{p}$ and (11), stacking all received samples corresponding to the pilot subcarriers gives (12), where $\mathbf{F}_{p}$ collects rows of $\mathbf{F}$ corresponding to the pilot subcarriers set $I_{p}$. In (12), we have separated the effect of data from pilots, and the terms with the unknown data and noise are contained in $\boldsymbol{\delta}$. Notice that, if the channel is slowly fading, due to the orthogonal property among subcarriers, the received signal on the $k^{t h}$ subcarrier $\bar{y}_{k}$ is completely dependent on $x_{k}$ and the corresponding mirror component $x_{N-k}$. In this situation, with the current pilot pair structure, channel estimation based on (12) is free of interference from the unknown data, and $\boldsymbol{\delta}$ is dependent only on noise. However, over DSCs, $\delta$ is dependent on both data and noise, which makes channel estimation suffer from interference caused by the unknown data. Fortunately, due to the fact that most of the ICI on one subcarrier comes from the neighboring subcarriers [6] [14], the current pilot cluster structure protects pilots in the middle of the cluster, and effectively reduces the effect of interference from the unknown data.

Writing the summation in (12) into matrix form gives

$$
\overline{\mathbf{y}}_{p}=\boldsymbol{\Theta}\left[\mathbf{E}_{p} \mathbf{x}_{p}\right] \mathbf{u}+\boldsymbol{\delta}
$$

where

$$
\begin{aligned}
\boldsymbol{\Theta}\left[\mathbf{E}_{p} \mathbf{x}_{p}\right] & =\left[\mathbf{F}_{p} \boldsymbol{\Phi}(-\rho) \mathbf{F}^{H} \operatorname{diag}\left\{\mathbf{E}_{p} \mathbf{x}_{p}\right\} \mathbf{F}_{L_{e}}, \cdots,\right. \\
& \mathbf{F}_{p} \boldsymbol{\Phi}(\rho) \mathbf{F}^{H} \operatorname{diag}\left\{\mathbf{E}_{p} \mathbf{x}_{p}\right\} \mathbf{F}_{L_{e}} \\
& \mathbf{F}_{p} \boldsymbol{\Phi}(-\rho) \mathbf{F}^{H} \operatorname{diag}\left\{\left(\mathbf{E}_{p} \mathbf{x}_{p}\right)^{\#}\right\} \mathbf{F}_{L_{e}}, \cdots \\
& \left.\mathbf{F}_{p} \boldsymbol{\Phi}(\rho) \mathbf{F}^{H} \operatorname{diag}\left\{\left(\mathbf{E}_{p} \mathbf{x}_{p}\right)^{\#}\right\} \mathbf{F}_{L_{e}}\right]
\end{aligned}
$$

and

$$
\mathbf{u}=\left[\mathbf{u}_{-\rho 1}^{T}, \cdots, \mathbf{u}_{\rho 1}^{T}, \mathbf{u}_{-\rho 2}^{T}, \cdots, \mathbf{u}_{\rho 2}^{T}\right]^{T}
$$

with length of $2(2 \rho+1) L_{e}$. By treating $\boldsymbol{\delta}$ as noise, the LS channel estimator is given by

$$
\hat{\mathbf{u}}=\left(\boldsymbol{\Theta}^{H}\left[\mathbf{E}_{p} \mathbf{x}_{p}\right] \Theta\left[\mathbf{E}_{p} \mathbf{x}_{p}\right]\right)^{-1} \Theta^{H}\left[\mathbf{E}_{p} \mathbf{x}_{p}\right] \overline{\mathbf{y}}_{p} .
$$

\section{B. Joint IQ imbalance and channel compensation}

In order to compensate for the IQ imbalance and channel so that the unknown data can be recovered, we substitute $\mathbf{x}=$ $\mathbf{E}_{d} \mathbf{x}_{d}+\mathbf{E}_{p} \mathbf{x}_{p}$ and $\mathbf{x}^{\#}=\mathbf{F F}\left(\mathbf{E}_{d} \mathbf{x}_{d}^{*}+\mathbf{E}_{p} \mathbf{x}_{p}^{*}\right)$ into (10), and the expression becomes (17), as shown at the top of this page, where the unknown data are in the first two terms only.

Considering the effects of both DSC and IQ imbalance, the received frequency domain signals on the subcarrier sets $[n-\beta, \cdots, n+\beta]$ and $[N-(n-\beta), \cdots, N-(n+\beta)]$ are selected to detect the data on the $n^{t h}$ subcarrier. Based on (17), we have

$$
\mathbf{f}_{n} \triangleq \overline{\mathbf{y}}_{n}-\boldsymbol{\eta}_{n}[\mathbf{u}]=\mathbf{B}_{1}^{n}[\hat{\mathbf{u}}] \mathbf{x}_{d}+\mathbf{B}_{2}^{n}[\hat{\mathbf{u}}] \mathbf{x}_{d}^{*}+\overline{\mathbf{w}}_{n}
$$

where $\overline{\mathbf{y}}_{n}, \boldsymbol{\eta}_{n}[\mathbf{u}], \mathbf{B}_{1}^{n}, \mathbf{B}_{2}^{n}$ and $\overline{\mathbf{w}}_{n}$ collect rows of $\overline{\mathbf{y}}, \boldsymbol{\eta}[\mathbf{u}]$, $\mathbf{B}_{1}, \mathbf{B}_{2}$ and $\overline{\mathbf{w}}$ corresponding to the sets $[n-\beta, \cdots, n+\beta]$ and $[N-(n-\beta), \cdots, N-(n+\beta)]$ respectively. Splitting the real and imaginary terms of the complex equation in (18) gives

$$
\left[\begin{array}{c}
\Re\left\{\mathbf{f}_{n}\right\} \\
\Im\left\{\mathbf{f}_{n}\right\}
\end{array}\right]=\mathbf{\Psi}\left[\begin{array}{l}
\Re\left\{\mathbf{x}_{d}\right\} \\
\Im\left\{\mathbf{x}_{d}\right\}
\end{array}\right]+\left[\begin{array}{l}
\Re\left\{\overline{\mathbf{w}}_{n}\right\} \\
\Im\left\{\overline{\mathbf{w}}_{n}\right\}
\end{array}\right]
$$

where

$$
\boldsymbol{\Psi}=\left[\begin{array}{cc}
\Re\left\{\mathbf{B}_{1}^{n}[\hat{\mathbf{u}}]\right\} & -\Im\left\{\mathbf{B}_{1}^{n}[\hat{\mathbf{u}}]\right\} \\
\Im\left\{\mathbf{B}_{1}^{n}[\hat{\mathbf{u}}]\right\} & \Re\left\{\mathbf{B}_{1}^{n}[\hat{\mathbf{u}}]\right\}
\end{array}\right]+\left[\begin{array}{cc}
\Re\left\{\mathbf{B}_{2}^{n}[\hat{\mathbf{u}}]\right\} & \Im\left\{\mathbf{B}_{2}^{n}[\hat{\mathbf{u}}]\right\} \\
\Im\left\{\mathbf{B}_{2}^{n}[\hat{\mathbf{u}}]\right\} & -\Re\left\{\mathbf{B}_{2}^{n}[\hat{\mathbf{u}}]\right\}
\end{array}\right] .
$$

Assuming the noise to be white with zero mean and power $\sigma_{\bar{w}}^{2}$, an approximated linear minimum mean square error (LMMSE) solution is

$$
\begin{aligned}
& {\left[\Re\left\{\hat{x}_{n}\right\}, \Re\left\{\hat{x}_{N-n}\right\}, \Im\left\{\hat{x}_{n}\right\}, \Im\left\{\hat{x}_{N-n}\right\}\right]^{T}} \\
& =\boldsymbol{\Psi}_{n}^{H}\left(\boldsymbol{\Psi} \boldsymbol{\Psi}^{H}+\frac{\sigma_{\bar{w}}^{2}}{\sigma_{x}^{2}} \mathbf{I}_{N_{d}}\right)^{-1} \mathbf{f}_{n}
\end{aligned}
$$

where $\boldsymbol{\Psi}_{n}$ collects columns of $\boldsymbol{\Psi}$ corresponding to $\left[\Re\left\{x_{n}\right\}, \Re\left\{x_{N-n}\right\}, \Im\left\{x_{n}\right\}, \Im\left\{x_{N-n}\right\}\right]$ and $\sigma_{x}^{2}$ is the energy 
of data. Notice that, $\sigma_{\bar{w}}^{2}$ is not the original white noise, but coupled with the IQ imbalance parameters, which is by no means available. For simplicity, by considering high SNRs, the data estimate is given by

$$
\left[\Re\left\{\hat{x}_{n}\right\}, \Re\left\{\hat{x}_{N-n}\right\}, \Im\left\{\hat{x}_{n}\right\}, \Im\left\{\hat{x}_{N-n}\right\}\right]^{T}=\boldsymbol{\Psi}_{n}^{H}\left(\boldsymbol{\Psi} \boldsymbol{\Psi}^{H}\right)^{-1} \mathbf{f}_{n},
$$

which is referred as the proposed compensator.

\section{SIMULATION RESULTS AND DISCUSSION}

In this section, computer simulation results are presented to demonstrate the performance of the proposed joint IQ imbalance and channel compensation algorithms. In the simulations, each OFDM symbol has 256 subcarriers $(N=256)$ and the length of $\mathrm{CP}$ is $L_{c p}=8$. The carrier frequency is $f_{c}=2 \mathrm{GHz}$ and the sample interval is $T_{s}=1 \mu \mathrm{s}$. The normalized maximal Doppler shift is set to $N f_{d} T_{s}=0.15$ (corresponding to a speed of $316.4 \mathrm{~km} / \mathrm{hr}$ ) for illustration purpose. The corresponding oversampling factor $G$ is set to 6 . The channel has three taps $(L=3)$ with an exponential power delay profile. Each tap coefficient follows a complex Gaussian distribution and is assumed to experience the same $f_{d}$, and the time correlation of each tap coefficient follows Jakes' model. Fourteen pilot pairs are used and each pair contains two clusters. The pilot cluster follows the structure in [6], and more specifically, each cluster occupies three pilots (i.e., $\lambda_{1}=\lambda_{2}=1$ ) with only one non-zero pilot in the middle of the cluster. The non-zero pilots are generated as zero-mean complex Gaussian random variables with power $\lambda_{1}+\lambda_{2}+1$. We set $\beta=2$, which means five main diagonals are taken into account for compensation. Data on other subcarriers are modulated by quadrature phase-shift keying (QPSK). With this arrangement, roughly $32.81 \%$ of the subcarriers and also $32.81 \%$ of the total power are occupied by pilots. The severe IQ imbalance setting [14] is considered with the amplitude imbalance $\alpha_{T}=\alpha_{R}=0.5 \mathrm{~dB}$, the phase imbalance $\phi_{T}=\phi_{R}=5^{\circ}$, and the impulse responses $\left.\mathcal{F}^{-1}\left\{H_{R I}(f)\right\}\right|_{n=0,1,2}=\left.\mathcal{F}^{-1}\left\{H_{T I}(f)\right\}\right|_{n=0,1,2}=$ $[0.01,1,0.01]$ and $\left.\mathcal{F}^{-1}\left\{H_{R Q}(f)\right\}\right|_{n=0,1,2}=$ $\left.\mathcal{F}^{-1}\left\{H_{T Q}(f)\right\}\right|_{n=0,1,2}=[0.01,1,0.02]$, respectively. Thus the front-end filter impulse response length is $L_{t}=L_{r}=3$. Each point in the following figures is obtained by averaging over 10,000 runs.

Fig. 2 shows the SER performance of the proposed compensation scheme with $N f_{d} T_{s}=0.15$. Here, 'without compensation' denotes the case without considering the IQ imbalance and the time-variation of the channel. The ideal case which assumes perfect IQ imbalance and channel state information is also depicted as a reference. The method in [14] is adopted for comparison. In the implementation of the method in [14], the pilot structure follows Scheme A described in [14], and five diagonals are considered for ICI compensation. For a fair comparison, within an OFDM symbol, the number of pilot subcarriers and the power allocated to pilots are the same as in our proposed algorithm. As can be seen, the proposed compensation scheme performs better than the method in [14].

\section{Conclusions}

In this paper, semi-blind IQ imbalance compensation for OFDM systems over doubly selective channels has been addressed. After reformulation of the system model, a joint IQ imbalance and channel estimation method has been developed, and the corresponding compensation scheme has also been proposed. Simulation results show that the proposed compensation method performs better than the method in [14].

\section{REFERENCES}

[1] C. Yeun, "Mobile TV technologies," in Proc. of IEEE International Conference on Information and Communications Techonology (ICICT), pp. 2-9, Cairo, Egypt, Dec. 2007.

[2] J. Zhou, Z. Ou, M. Rautiainen, T. Koskela and M. Ylianttila, "Digital television for mobile devices," IEEE Multimedia, vol. 16, no. 1, pp. 60-71, Jan.-March 2009.

[3] K.-T. LEE, "Technology leaders forum- create the future with mobile WIMAX," IEEE Communications Magazine, vol.45, no.5, pp. 10-14, May, 2007.

[4] X. Cai and G. B. Giannakis, "Bounding performance and suppressing intercarrier interference in wireless mobile OFDM," IEEE Trans. Commun., vol. 51, no. 12, pp. 2047-1056, Dec. 2003.

[5] I. Barhumi, G. Leus and M. Moonen, "Equalization for OFDM over doubly selective channels," IEEE Trans. Signal Processing, vol. 54, no. 4, pp. 1445-1457, Apr. 2006.

[6] Z. Tang, G. Leus, R.C. Cannizzaro and P. Banelli, "Pilot-assisted timevarying channel estimation for OFDM systems," IEEE Trans. Signal Processing, vol. 55, no. 5, pp. 2226-2238, May 2007.

[7] J. Tubbax etc., "Compensation of IQ imbalance and phase noise in OFDM systems," IEEE Trans. Wireless Communications, vol.4, no.3, pp. 872-877, May 2005.

[8] S. Tang etc., "Phase noise suppression in OFDM systems in presence of IQ imbalance," in Proc. of IEEE International Conference on Communications, Circuits and Systems (ICCCAS), pp. 1184-1188, Guilin, China, June 2006.

[9] Y. Kato, T. Ikuno and Y. Sanada, "IQ imbalance compensation scheme for MB-OFDM with transmit diversity," in Proc. of IEEE International Conference on Ultra-Wideband (ICUWB), pp. 293-298, Waltham, MA Sept. 2006.

[10] D. Tandur and M. Moonen, "Joint compensation of OFDM frequencyselective transmitter and receiver IQ Imbalance," EURASIP J. Wireless Communications and Networking, vol. 2007, no. 68563, pp. 1-10, 2007.

[11] P. Rykaczewski, M. Valkama and Markku Renfors, "On the connection of I/Q imbalance and channel equalization in direct-conversion transceivers," IEEE Trans. Vehicular Technology, vol. 57, no. 3, pp. 1630-1636, May 2008.

[12] H. Lin and K. Yamashita, "Frequency offset and I/Q imbalance compensation for direct-conversion receivers," IEEE Trans. Wireless Commun., vol. 8, no. 1, pp. 18-23, Jan. 2009.

[13] I. Lu and J.Chang, "Joint transmitter and receiver IQ imbalance estimation and compensation for OFDM systems," in Proc. of IEEE International Conference on Radio and Wireless Symposium (RWS), pp. 476-479, New Orleans, LA, Jan. 2010.

[14] B. Narasimhan, D. Wang, S. Narayanan, H. Minn and N. Al-Dhahir, "Digital compensation of frequency-dependent joint Tx/Rx I/Q imbalance in OFDM systems under high mobility," IEEE J. Select. Topics Signal Processing, vol. 3, no. 3, pp. 405-417, June 2009.



Fig. 2. SER performance of the proposed compensation scheme $\left(N f_{d} T_{s}=\right.$ $0.15)$. 